\title{
Logical Pluralism: Another Application for Chunk and Permeate
}

\author{
Graham Priest \\ Departments of Philosophy \\ Universities of Melbourne, St Andrews, and \\ the Graduate Center, City University of New York
}

9th March 2013

\begin{abstract}
A motivation behind one kind of logical pluralism is the thought that there are different kinds of objects, and that reasoning about situations involving these different kinds requires different kinds of logics. Given this picture, a natural question arises: what kind of logical apparatus is appropriate for situations which concern more than one kind of objects, such as may arise, for example, when considering the interactions between the different kinds? The paper articulates an answer to this question, deploying the methodology of Chunk and Permeate, developed in a different context by Brown and Priest (2004).
\end{abstract}

\section{Logical Pluralism and Crossing Kinds}

A popular motivation for logical pluralism is something like this: there are different kinds of situations, and different logics (or consequence relations) may be appropriate for reasoning about them - in the sense that if you know (or assume) that certain things hold in these situations, the logic is guaranteed to give you other things that hold in the situation. ${ }^{1}$ For example,

\footnotetext{
${ }^{1}$ The pluralisms of Batens (1985), (1990), Beall and Restall (2006), Bueno (2002), and da Costa (1997), though very different, can all be seen in this way. I do not suggest that any of them would endorse the following examples.
} 
one might hold that intuitionist logic is the appropriate logic for reasoning about a situation involving mathematical constructions; that quantum logic is appropriate for a situation involving quantum objects; that classical logic is appropriate for a situation involving medium-sized dry goods; that paraconsistent logic is appropriate for a situation involving (naive) inconsistent sets; and so on. Though I do not subscribe to this view myself, ${ }^{2}$ let us grant it here.

An obvious issue arises when it is observed that sometimes we may have to reason about a situation that concerns more than one kind of object-for example, in considering the relations between them. Thus, quantum states and observational devices (which are medium sized dry goods) interact. In reasoning about their interactions, we need to reason about both. Or again, one of the things that the naive notion of set applies to is mathematical constructions. To reason about this application, we need to reason about a situation involving both constructions and sets. Now, the question is: what logic is it appropriate to apply in such cases?

A natural thought is that we should apply the intersection of the logics appropriate for the different pure kinds. An inference is acceptable if it is valid in all the relevant logics. But this logic is likely to be too weak: in reasoning about different kinds of objects collectively, we will sometimes have to reason about each kind severally, and the intersection logic is liable to be too weak for this. Thus, for example, suppose that we find ourselves reasoning with a sentence of the form $P a \rightarrow a R b$, where $a$ and $b$ are of different kinds. We can use the logic common to things of $a$ 's kind and things of b's kind. Maybe this includes modus ponens. But we may need to reason about things of just $a$ 's kind to establish $P a$; and this may need more than just the logic in the intersection.

Another thought is that we need a logic which is sui generis. What such a logic would be like, and, indeed, whether one can construct an appropriate one at all, would be pressing questions. But there is something unsatisfactory about this approach anyway. Situations concerning the interface between different kinds of objects surely depend on the situations concerning those objects themselves. The appropriate logic for the situation is not, therefore, sui generis; it is, at least in part, a function of the logics for each kind.

The purpose of this paper is to suggest a more satisfactory answer to the question. I will give a model of how different logics may be combined in

\footnotetext{
${ }^{2}$ Priest (2006), ch. 12.
} 
a non-trivial fashion, allowing us to reason across kinds. The model is an application of the method chunk and permeate $(C \& P) .{ }^{3}$ In this, reasoning is partitioned into discrete "chunks"; but the chunks are not isolated: information of certain kinds is allowed to flow ("permeate") between the chunks. The model was developed to account for a certain kind of reasoning with inconsistent information. Reasoning in a certain way, we establish conclusions about various objects. The results of this reasoning are then fed into another theory, where we continue to reason, but on a basis different from, indeed, inconsistent with, the first. (The reasoning of the original infinitesimal calculus seems to be of this kind. In this, we establish properties of certain functions on the assumption that infinitesimals are non-zero. We then feed these results into another theory, and continue to reason, but on the assumption that infinitesimals are zero.) In the applications of the model already given, the reasoning in each chunk was classical; but, as was pointed out, ${ }^{4}$ there is absolutely no reason why this must be the case: the logic may vary from chunk to chunk. If this be the case, the model provides a natural way of handling the application of different logics in combination.

In Section 2, I will describe the general structure of a $C \& P$ model. In Section 3 we will look at some simple applications of the model.

\section{$2 \quad C \& P$ Structures}

The basic units of a $C \& P$ structure are the chunks. A chunk, $C_{i}$, is a theory. It has a language, $L_{i}$, with a set of closed formulas, $F_{i}$, a set of axioms, $A_{i}$, and a logical consequence relation, $\vdash_{i}$. The languages of chunks may be different, but may overlap. The machinery of permeability between the chunks $i$ and $j(i \neq j)$ comprises two parts: the permeability function, $\rho_{i j}$, which is a subset of $F_{i}$; and a translation function, $t_{i j}$, which is a map from $F_{i}$ to $F_{j}$. The map provides a way of translating the sentences of $L_{i}$ into those of $L_{j}{ }^{5} \quad$ (In the original papers, $t_{i j}$ was always the identity function, and so it received no separate mention.) Notation: if $\Sigma$ is a set of

\footnotetext{
${ }^{3}$ Brown and Priest (2004), (200+).

${ }^{4}$ Brown and Priest (2004), Section 5.

${ }^{5} \mathrm{~A}$ natural assumption is that $\rho_{i j}$ should be identified syntactically, so that it is recursive. All the examples in the paper are of this kind. But in principle anyway, it could be detemined in some other way. There is no reason in theory why is should not be a set of arbitrary computational complexity. Of course, if practice, not theory, is important, matters are different.
} 
sentences, then $\Sigma^{t}$ is just $\{t(\sigma): \sigma \in \Sigma\}$. A $C \& P$ structure then has the form $\mathfrak{S}=\left\langle\left\{\left\langle L_{i}, A_{i}, \vdash_{i}\right\rangle: i \in I\right\},\left\{\left\langle\rho_{i j}, t_{i j}\right\rangle: i, j \in I, i \neq j\right\}, o\right\rangle . o$ is a distinguished member of $I$, and $C_{o}$ is the output chunk.

Intuitively, the structure operates as follows. We compute the consequences of each chunk in the standard way. For each $i \in I$, the consequences of the chunk $C_{i}$ that are in $\rho_{i j}$-or, more precisely, their translations - are them added to $A_{j}$. We then compute the consequences of each chunk anew, and repeat the process. The final output of each chunk is what results after we have been through this process $\omega$ times, and the output of the structure is the output of $C_{o}{ }^{6}$

More formally, the axioms, $A_{i}^{n}$, and theorems, $T_{i}^{n}$, of chunk $i$ at the $n$th stage of the process are defined by a joint recursion on $n$ as follows:

$$
\begin{aligned}
& A_{i}^{0}=A_{i} \\
& T_{i}^{0}=\left\{\alpha: A_{i}^{0} \vdash_{i} \alpha\right\} \\
& A_{i}^{n+1}=A_{i}^{n} \cup \bigcup_{i \neq j \in I}\left(T_{j}^{n} \cap \rho_{j i}\right)^{t_{j i}} \\
& T_{i}^{n+1}=\left\{\alpha: A_{i}^{n+1} \vdash_{i} \alpha\right\}
\end{aligned}
$$

The eventual output of each chunk, $T_{i}^{\omega}$, is defined in the obvious way: $T_{i}^{\omega}=$ $\bigcup_{n \in \omega} T$. And $\mathfrak{S} \Vdash \alpha$ iff $\alpha \in T_{o}^{\omega}$.

Such is the general structure. It is much more powerful than is necessary for most applications. In particular, if there is only a finite number of chunks, and there are no cycles in the flow of information between chunks, then the final output of each chunk is established after some finite $n$. Such will be the case is what follows.

Before we move on to some examples, I note that the whole $C \& P$ mechanism can be formulated as one "hyper-theory". The language of the theory is the language of $C_{o}$, augmented, for every $i \in I$, by a monadic predicate, $T_{i}$, and a name $\langle\alpha\rangle$, for every formula, $\alpha$, of $L_{i}$. $T_{i}\langle\alpha\rangle$ means, intuitively, that $\alpha$ is a theorem of chunk $i$. The axioms are:

- for each $i \in I$, and $\alpha \in A_{i}, T_{i}\langle\alpha\rangle$

The logic of the theory is $\vdash_{o}$, augmented by the rules of inference:

\footnotetext{
${ }^{6}$ And obviously, if this output is to be more than just the consequences of $A_{0}$, some information had better flow into $C_{0}$ !
} 
- for every $i \in I$, and every valid inference, $\alpha_{1}, \ldots, \alpha_{n} \vdash_{i} \beta, T_{i}\left\langle\alpha_{1}\right\rangle, \ldots, T_{i}\left\langle\alpha_{n}\right\rangle \vdash$ $T_{i}\langle\beta\rangle$

- for every $\alpha$ in $\rho_{i j}$, and $i \neq j \in I, T_{i}\langle\alpha\rangle \vdash T_{j}\left\langle t_{i j}(\alpha)\right\rangle$

- for every $\alpha$ in $L_{o}, T_{o}\langle\alpha\rangle \vdash \alpha$.

A little thought suffices to show that any proof in the $C \& P$ machinery can be mirrored in this single hyper-theory.

\section{$3 \quad$ Examples}

I will now give four examples of the $C \& P$ mechanism to show how it handles the use of a plurality of consequence relations.

\subsection{Example 1: Different Logics for Different Kinds of Objects}

For the first example, we suppose that we have two different kinds of object, that different logics are appropriate for reasoning about the different kinds, but that we sometimes wish to reason about both kinds of object together.

There are three chunks. $C_{1}$ is a theory of objects of kind $1 . L_{1}$ is a firstorder language with constants in the set $\mathcal{C}_{1}$, and predicates in the set $\mathcal{P}_{1}$. The theory of $C_{1}$ has an underlying logic, $\vdash_{1}$, say intuitionist logic, and some appropriate set of axioms. $C_{2}$ is a theory of objects of kind 2. The language is a first-order language with constants in the set $\mathcal{C}_{2}$, and predicates in the set $\mathcal{P}_{2}$ (where $\mathcal{C}_{1} \cap \mathcal{C}_{2}=\phi=\mathcal{P}_{1} \cap \mathcal{P}_{2}$ ). $C_{2}$ has an underlying logic, $\vdash_{2}$, say some paraconsistent logic, and some appropriate set of axioms. $C_{0}$ is the output chunk. $L_{0}$ is a first-order language whose vocabulary contains the union of those of $L_{1}$ and $L_{2}$, but also contains other predicates. In particular, it has two new monadic predicates, $K_{1}$ and $K_{2}$. (Intuitively, these are going to apply to objects of kinds 1 and 2, respectively.) Its axioms concern the predicates particular to the language, and its logic is the intersection of $\vdash_{1}$ and $\vdash_{2}$; that is, $\Sigma \vdash_{0} \alpha$ iff $\Sigma \vdash_{1} \alpha$ and $\Sigma \vdash_{2} \alpha . \rho_{10}=F_{1}$, and $t_{10}$ is the identity translation, except that all quantifiers are relativised to $K_{1}$. That is, sentences of the form $\exists x \alpha$ and $\forall x \alpha$ are translated as $\exists x\left(K_{1} x \wedge \alpha\right)$ and 
$\forall x\left(K_{1} x \rightarrow \alpha\right)$ respectively. ${ }^{7} \rho_{20}$ and $t_{20}$ are exactly the same, except that ' 1 ' is replaced by ' 2 '. For all other $i \neq j, \rho_{i j}=\phi$. We may depict the structure as follows:

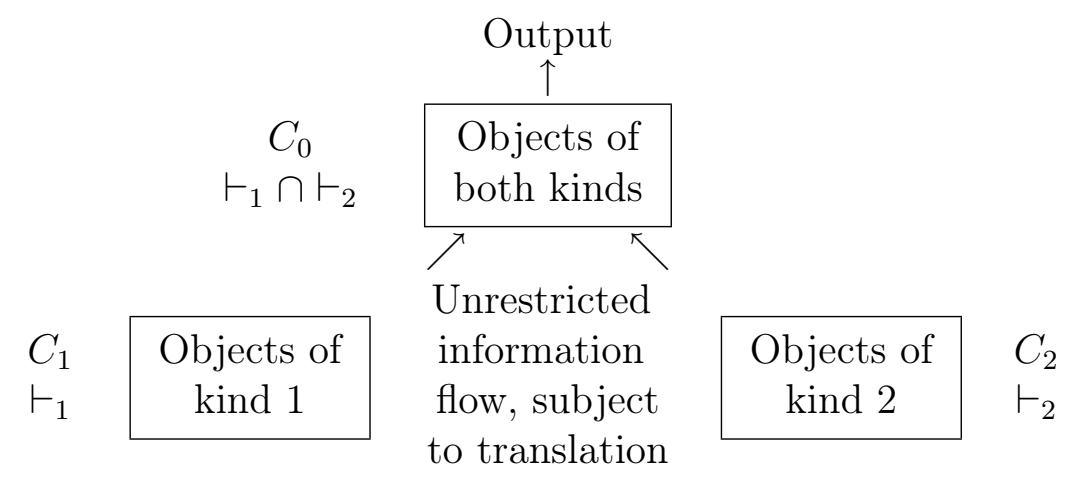

Reasoning works as follows. Within $C_{1}$ we may reason about objects of kind 1 , according to $\vdash_{1}$. Results permeate into $C_{0}$ (in translated form), and since this is the output chunk, are outputted. Similarly for objects of kind 2 and $C_{2}$. But, using the information established in $C_{1}$ and $C_{2}$, and any other information provided by the axioms of $C_{0}$, we may reason about both kinds of objects and their relations. Whether or not new facts about objects of just kind 1 may be established in the process depends on the axioms of $C_{0}$. If they can, they cannot be inferred from the information that permeates into $C_{0}$ from $C_{1}$ alone, since $\vdash_{0}$ is a sub-logic of $\vdash_{1}$. Similarly for objects of kind 2 .

\subsection{Example 2: Consistent Objects within an Inconsis- tent Theory}

The second example concerns how one may reason about consistent objects in a context where not everything behaves consistently. Essentially, one would like to apply classical logic to, but only to, such objects. To illustrate, let us suppose that numbers behave consistently, but sets, in general, do not.

There are two chunks, $C_{N}$ and $C_{S}$. The output chunk is $C_{S} . L_{N}$ is the language of first-order arithmetic: one constant, 0 , two binary predicates, $=$ and $S$ (successor), and the usual quantifiers and connectives. (The example could be extended to allow the language to contain the predicates of addition

\footnotetext{
${ }^{7}$ Possibly, other translations might be appropriate. Thus, ' $\rightarrow$ ' here might be replaced by ' $\supset$ ', where $A \supset B$ is $\neg A \vee B$.
} 
and multiplication as well; but let us keep things simple.) The axioms of $C_{N}$ are the Peano axioms (say), and the logic is classical. $L_{S}$ is the language of a first-order relevant logic, with two binary predicates, $=$ and $\epsilon$, and setabstracts. I write the conditional connective as $\rightarrow ; \alpha \supset \beta$ is defined as $\neg \alpha \vee \beta$. The axioms of $C_{S}$ are those of naive set theory:

- $x \in\{y: \alpha(y)\} \leftrightarrow \alpha(x) \quad$ (for every $\alpha$ )

- $\forall x(x \in y \leftrightarrow x \in z) \rightarrow y=z$

The underlying logic of $C_{S}$ is an appropriate relevant logic, which makes the axioms inconsistent but non-trivial. ${ }^{8}$

$\rho_{S N}=\phi . \rho_{N S}=F_{N}$, and $t_{N S}$ is the natural translation. In particular:

- 0 is translated into $\{x: \neg \exists y x \in y\}$

- $S x y$ is translated into $y=x \cup\{x\}$

Connectives remain themselves (so that $\supset$ is translated into $\supset$ ), and quantifiers are restricted to $\omega$, defined as:

- $\{z: 0 \in z \wedge \forall x \forall y((x \in z \wedge S x y) \rightarrow y \in z)\}$

so that $\exists x \alpha$ and $\forall x \alpha$ are translated as $\exists x(x \in \omega \wedge \alpha)$ and $\forall x(x \in \omega \supset \alpha)$, respectively. ${ }^{9}$ We may depict the structure as follows:

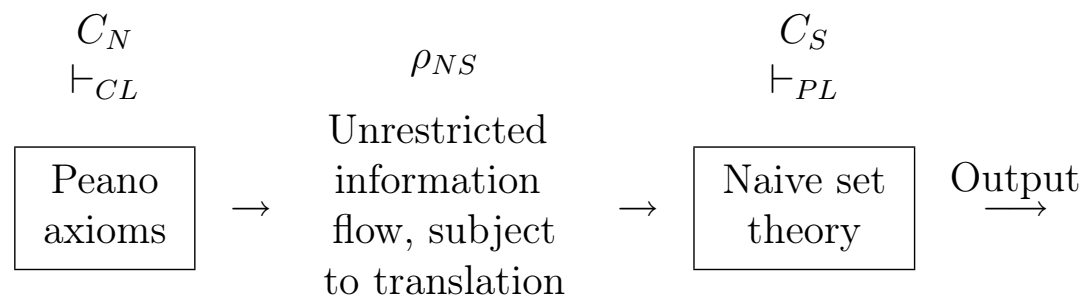

The output contains all the theorems of naive set theory. Given the standard definitions of numbers, the logic of $C_{S}$ will be too weak to prove all the theorems of Peano Arithmetic. However, these may be proved in $C_{N}$ and then allowed to permeate into $C_{S}$. These theorems, then, are not only part of the eventual output, but may be appealed to in $C_{S}$ to establish results about sets that involve natural numbers.

\footnotetext{
${ }^{8}$ See Priest (1987), ch. 10, and Priest (2002), Section 8.

${ }^{9}$ I assume that these are the natural translations. They are, after all, what the restricted quantifiers express in their original classical context. But I could imagine situations where the ' $\supset$ ' should be replaced by ' $\rightarrow$ ', or even some entirely different conditional, such as that of Beall, Brady, Hazen, Priest, and Restall (2006).
} 


\subsection{Example 3: Obtaining Observation Data from a Theory}

In the previous two examples, the output chunk had a logic weaker than the chunks which delivered information into it. In the next two examples, the output chunk has a stronger logic. Suppose that one has a scientific theory couched in language that is partly observational and partly theoretical. Suppose that the theory uses some non-classical logic, say quantum logic, but that one wants to use its observable predictions, and, moreover, to reason classically using them. Then one may allow these to permeate into a classical output chunk.

More specifically, let there be two chunks, $C_{T}$ and $C_{O} . C_{O}$ is the output chunk. $L_{T}$ is a first-order language. We suppose that its constants are divided up (disjointly) into the observational, $\mathcal{C}_{O}$, and theoretical $\mathcal{C}_{T}$. Similarly, its predicates are divided up (disjointly) into the observational, $\mathcal{P}_{O}$, and theoretical $\mathcal{P}_{T}$. The observational sentences, $\mathcal{O}$, are the atomic sentences formed from $\mathcal{C}_{O}$ and $\mathcal{P}_{O}$, together with their negations. $C_{T}$ has certain axioms, and an underlying quantum logic. $C_{O}$ is a first-order language which contains only the constants $\mathcal{C}_{O}$ and predicates $\mathcal{P}_{O}$. It has axioms that involve these, and the underlying logic is classical. $\rho_{O T}=\phi . \rho_{T O}=\mathcal{O}$, and $t_{T O}$ is the identity translation. So the set-up is as follows:

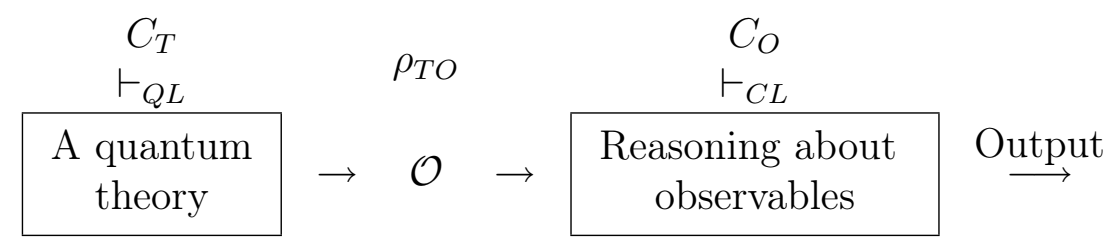

Whether we interpret the quantum theory realistically or instrumentally, the structure allows us to make use of its observable predictions, reasoning about them classically in the output chunk.

\subsection{Example 4: Extracting Consistent Information from an Inconsistent Theory}

In the final example, we have an inconsistent theory, and the $C \& P$ mechanism is used to extract consistent information, on the basis of which we may then reason classically. Thus, suppose that we have an inconsistent theory of truth, based on some paraconsistent logic. This will tell us that some 
things of the form $T\langle\alpha\rangle$ and $T\langle\neg \alpha\rangle$ hold (where $T$ is the truth predicate and $\langle$.$\rangle is some name-forming device). Suppose, however, that we think that$ statements of the form $T\langle\alpha\rangle$ are themselves consistent. We can allow them to permeate into another chunk, where we can reason classically about them.

More specifically, let there be two chunks, $C_{P}$ and $C_{C}$. $C_{C}$ is the output chunk. $C_{P}$ is a naive theory of truth, with the $T$-schema and self-reference. It is inconsistent (for example, if $\lambda$ is a liar sentence, $\neg T\langle\lambda\rangle, C_{P}$ can prove $T\langle\lambda\rangle, \neg T\langle\lambda\rangle$, and $T\langle\neg \lambda\rangle$ ), but non-trivial. ${ }^{10} L_{C}$ is the same as $L_{P}$, but its axioms are the empty set. (In particular, it does not contain the $T$-schema.) Its underlying logic is classical. $\rho_{C P}=\phi . \rho_{P C}$ is the set of sentences of the form $T\langle\alpha\rangle$, where $\alpha$ is a sentence of the language, and $t_{P C}$ is the identity map. ${ }^{11}$ So the setup is as follows:

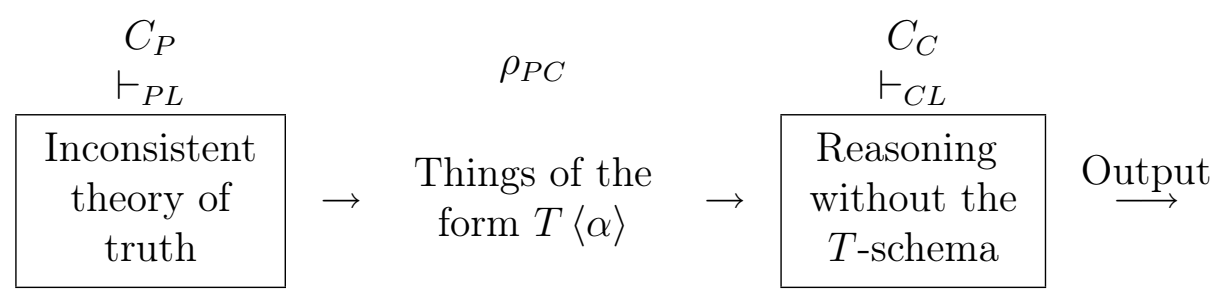

The structure allows us to make use of a consistent part of the output of the inconsistent theory or truth, but then to go on and reason classically using it.

\section{Conclusion}

The four examples illustrate some of the possible applications of $C \& P$ to reasoning that requires logical pluralism. The $C \& P$ structures involved are all fairly simple. For example, information flow is only ever one-way in them. But they suffice to show how the structure makes possible reasoning that employs more than one logic, and in a sensible, controlled, way. ${ }^{12}$

\footnotetext{
${ }^{10}$ See Priest (1987), ch. 9, and Priest (2002), Section 8.

${ }^{11} \mathrm{~A}$ somewhat different permeability relation could allow through all the consistent consequences of $C_{P}$ (i.e., all those provable $\alpha$ for which $\neg \alpha$ cannot also be proved). In general, though, this would make the filter highly non-effective.

${ }^{12}$ Versions of this paper were given in 2008 at a meeting of the Melbourne Logic Group, and at the conference Logical Pluralism in Tartu, Estlonia. I'm grateful for the helpful comments of the members of those audiences. Thanks, too, go to an anonymous referee for this volume.
} 


\section{References}

[1] Batens, D. (1985), 'Meaning, Acceptance and Dialectics', pp. 333-60 of J. C. Pitt (ed.), Change and Progress in Modern Science, Dordrecht: Reidel.

[2] Batens, D. (1990), 'Against Global Paraconsistency', Studies in Soviet Thought, 39: 209-29.

[3] Beall, JC, Brady, R., Hazen, A., Priest, G., and Restall, G. (2006), 'Relevant Restricted Quantification', Journal of Philosophical Logic 35: $587-98$.

[4] Beall, JC, and Restall, G. (2006), Logical Pluralism, Oxford: Oxford University Press.

[5] Brown, B., and Priest, G. (2004), 'Chunk and Permeate I: the Infinitesimal Calculus', Journal of Philosophical Logic 33: 379-88.

[6] Brown, B., and Priest, G. $(200+)$, 'Chunk and Permeate II: the Bohr Theory of the Atom', to appear.

[7] Bueno, O. (2002), 'Can a Paraconsistent Theorist be a Logical Monist?', ch. 29 of W. Carnielli, M. Coniglio, and I. M. L. D'Ottaviano (eds.), Paraconsistent Logic: the Logical Way to be Inconsistent, New York, NY: Marcel Dekker.

[8] da Costa, N. C. A. (1997), Logique Classique et Non-Classique: Essai sur les Fondements de la Logique, Paris: Masson.

[9] Priest, G. (1987), In Contradiction: a Study of the Transconsistent, Dordrecht: Martinus Nijhoff; second edition, Oxford: Oxford University Press, 2006.

[10] Priest, G. (2002), 'Paraconsistent Logic', pp. 287-393, Vol. 6, of D. Gabbay and F. Guenthner (eds.), Handbook of Philosophical Logic, 2nd edition, Dordrecht: Kluwer Academic Publishers.

[11] Priest, G. (2006), Doubt Truth to be a Liar, Oxford: Oxford University Press. 


\section{University Library}

\section{- M M N E R VA A gateway to Melbourne's research publications}

Minerva Access is the Institutional Repository of The University of Melbourne

Author/s:

Priest, $\mathrm{G}$

Title:

Logical Pluralism: Another Application for Chunk and Permeate

Date:

2014

Citation:

Priest, G. (2014). Logical Pluralism: Another Application for Chunk and Permeate.

Erkenntnis: an international journal of analytic philosophy, 79 (SUPPL.2), pp.331-338. https:// doi.org/10.1007/s10670-013-9472-1.

Persistent Link:

http://hdl.handle.net/11343/282925 